\title{
Germinação em temperatura sub-ótima de embriões de Inga vera subsp. affinis obtidos sob diferentes condições ambientais
}

Germination at suboptimal temperature of embryos of Inga vera subsp. affinis produced at different environmental conditions

\author{
Edmir Vicente Lamarca ${ }^{1}$, Marcio Roberto Bonjovani ${ }^{2}$, José Marcio Rocha Faria ${ }^{3}$ \& Claudio José Barbedo ${ }^{4,5}$
}

\begin{abstract}
Resumo
As condições hídricas e térmicas do ambiente durante a formação das sementes podem condicionar diversas respostas fisiológicas, como a exigência térmica para a germinação. Neste trabalho analisou-se a germinação de embriões de Inga vera subsp. affinis de origens distintas em resposta a diferentes temperaturas, incluindo-se o cálculo dos graus-dia e da chuva acumulada durante o desenvolvimento e maturação. Os embriões foram obtidos de frutos maduros de matrizes localizadas em São Paulo e Minas Gerais e analisados quanto ao teor de água, conteúdo de massa seca e germinação. Os resultados demonstram variações nas respostas germinativas entre as origens dos materiais, sob temperatura sub-ótima. Dependendo da origem os embriões de I. vera subsp. affinis germinam a partir de $10^{\circ} \mathrm{C}$ ou $15^{\circ} \mathrm{C}$ e apresentam melhor desempenho em tempo a partir de $20^{\circ} \mathrm{C}$ ou $25^{\circ} \mathrm{C}$. Tais variações podem estar associadas, entre outros fatores, às condições do ambiente de formação e ao grau de maturidade das sementes no momento da dispersão.
\end{abstract}

Palavras-chave: graus-dia, sementes florestais, Leguminosae.

\begin{abstract}
Physiological responses like the thermal requirements for germination may be influenced during seed formation by environmental conditions such as water availability and temperature. Here we analyzed germination of embryos of Inga vera subsp. affinis from different sources in response to various temperature regimes, including calculation of degree days and accumulated rainfall during development and maturation. Embryos were taken from ripe fruits collected on trees located in São Paulo and Minas Gerais, and were analyzed as to germination, water content and dry matter content. The results showed that the origin of the embryos was related to variation in response under sub-optimal temperatures. Depending on the origin, I. vera subsp. affinis embryos were able to germinate even at low temperatures $\left(10\right.$ to $\left.15^{\circ} \mathrm{C}\right)$, with better performance attained from 20 to $25^{\circ} \mathrm{C}$. This variation may be associated, among other factors, to environmental conditions during maturation and to the maturation stage at seed dispersal.
\end{abstract}

Key words: degree-day, forest seeds, Leguminosae.

\section{Introdução}

A germinação de sementes é dependente de numerosos fatores abióticos, dentre os quais a temperatura é um dos mais importantes, sendo relevante seu entendimento nas temperaturas ótimas ou extremas. Sementes de espécies florestais tropicais e subtropicais do Brasil germinam em ampla faixa térmica e parecem apresentar relação positiva entre a temperatura ótima e o regime térmico da região de sua ocorrência natural (Borghetti 2005). Frequentemente, a faixa térmica adequada para a germinação de sementes dessas espécies está entre 20 e $30^{\circ} \mathrm{C}$ (Mello \& Barbedo 2007; Stockman et al. 2007; Pires et

\footnotetext{
${ }^{1}$ Instituto de Botânica, Programa de Pós-Graduação em Biodiversidade Vegetal e Meio Ambiente, Av. Miguel Stéfano 3687, 04301-012, São Paulo, SP, Brasil. lamarcabio@ig.com.br

${ }^{2}$ Universidade Estadual Paulista (Unesp), Inst. Biociências, Programa de Pós-Graduação em Ciências Biológicas, Distrito de Rubião Jr. s/n ${ }^{\circ}, 18618-970$, Botucatu, SP. bonjovanimr@hotmail.com

${ }^{3}$ Universidade Federal de Lavras, Depto. Ciências Florestais, C.P. 3037, 37200-000, Lavras, MG, Brasil. jmfaria@dcf.ufla.br

${ }^{4}$ Instituto de Botânica, Núcleo de Pesquisa em Sementes. claudio.barbedo@pesquisador.cnpq.br

${ }^{5}$ Autor para correspondência: claudio.barbedo@pesquisador.cnpq.br
} 
al. 2009; Pimenta et al. 2010; Nascimento et al. 2011; Lamarca et al. 2011; Pascuali et al. 2012). Contudo, podem ocorrer variações até mesmo entre populações da mesma espécie, em decorrência das condições ambientais e das características adaptativas e ecológicas (Daws et al. 2004; Lamarca et al. 2011; Mattana et al. 2012).

Variações na temperatura do ar durante o desenvolvimento e maturação podem condicionar diversas respostas fisiológicas nas sementes formadas, como a exigência térmica e a temperatura basal para a germinação. Contudo, as variações no comportamento germinativo entre diferentes sementes de uma mesma espécie podem ser decorrentes, também, do estádio de maturação em que a semente se encontrava quando foi colhida ou dispersa. A variação na tolerância à dessecação de sementes de uma mesma espécie, por exemplo, pode ser influenciada pelas condições nas quais foram formadas, produzindo sementes de maior ou menor grau de maturação (Daws et al. 2004, 2006; Delgado \& Barbedo 2012). Por outro lado, essa variação no grau de maturação também pode gerar diferenças na exigência térmica para a germinação (Lamarca et al. 2011). Essas variações podem ser mensuradas e expressas em graus-dia (Daws et al. 2004, 2006).

Popularmente conhecida por ingá, ingádo-brejo ou ingazeiro, Inga vera Willd. subsp. affinis (DC.) T.D. Pennington pertence à família Leguminosae e está distribuída na América tropical e subtropical, na Mata Atlântica e em áreas de vegetação ripária (Joly 1993; Bilia et al. 1998). Suas sementes podem ser utilizadas como um modelo para estudos fisiológicos, por serem intolerantes à dessecação e terem curtíssima longevidade (Bilia et al. 2003). Além disso, conta com diversas informações científicas, principalmente as referentes ao armazenamento e à tolerância à dessecação (Bilia et al. 1998, 1999; Barbedo \& Cicero 2000; Faria et al. 2004; Andréo et al. 2006; Bonjovani \& Barbedo 2008; Parisi et al. 2013). A temperatura ótima para a germinação dessas sementes, que frequentemente está associada com as condições ambientais da região de origem da espécie, está entre 25 e $30^{\circ} \mathrm{C}$ (Bilia \& Barbedo 1997), mas não há estudos comparando sementes de diferentes regiões. Portanto, a análise do comportamento germinativo das sementes de diferentes regiões pode fornecer importantes informações quanto ao grau de interferência do ambiente sobre as características das sementes formadas. A germinação em condições sub-ótimas de temperatura, por exemplo, permite analisar o grau de plasticidade desenvolvido pelas diferentes populações da mesma espécie, conforme as condições ambientais nas quais cada uma foi selecionada. Neste trabalho, essa plasticidade foi analisada pela germinação de embriões de $I$. vera subsp. affinis de origens distintas.

\section{Material e Métodos}

As sementes de $I$. vera subsp. affinis foram obtidas de frutos maduros, baseando-se nas informações de maturação e colheita de Figliolia \& Kageyama (1994), Barbedo \& Cicero (2000) e Bonjovani \& Barbedo (2008), das seguintes regiões: Ribeirão Vermelho (RIB), Piracicaba (PIR), Vinhedo (VIN), Pariquera-açú (PAR) e São Paulo (SAP). Desta última, foram obtidos frutos em três anos: 2007 (SAP2007), 2008 (SAP2008) e 2010 (SAP2010). As coordenadas geográficas desses locais estão apresentadas na Tabela 1.

Dados diários de precipitação pluvial e temperatura (máxima e mínima) do ar, respectivamente, para o cálculo da chuva acumulada e dos graus-dia, do período compreendido entre o máximo florescimento dos indivíduos e a colheita dos frutos maduros, foram obtidos de estações meteorológicas localizadas próximas às áreas de coleta (disponibilizados pela Universidade Federal de Lavras, pelo Instituto Agronômico de Campinas e pelo Instituto Astronômico e Geofísico da Universidade de São Paulo). O somatório de graus-dia (GD), da antese à colheita, foi calculado segundo equações propostas por Villa Nova et al. (1972), considerando a temperatura base de $10^{\circ} \mathrm{C}$ (Pedro Junior et al. 1977). Esses dados também estão apresentados na Tabela 1.

Após a colheita, os frutos foram abertos manualmente para retira das sementes. A seguir, removeu-se o tegumento (sarcotesta), obtendo-se os embriões utilizados nos experimentos. Após esse procedimento, os embriões foram armazenados em câmara fria a $7^{\circ} \mathrm{C}$ até o início dos experimentos, não excedendo sete dias após a colheita, de acordo com recomendação de Bilia et al. (1998).

Os embriões foram caracterizados quanto ao teor de água, conteúdo de massa seca e germinação. O teor de água e a massa seca foram determinados gravimetricamente, pelo método de estufa a $103^{\circ} \mathrm{C}$ por 17 horas (Brasil 2009) e os resultados apresentados, respectivamente, em $\mathrm{g}$ de água $\cdot \mathrm{g}$ de massa seca ${ }^{-1}\left(\mathrm{~g} \mathrm{~g}^{-1}\right)$ e g embrião ${ }^{-1}$. 
Tabela 1 - Origem de embriões de Inga vera subsp. affinis. Localização geográfica, classificação climática de Köppen, período de maturação e dados meteorológicos, compreendido entre o florescimento e colheita (média da temperatura máxima e mínima do ar, graus-dia e chuva acumulada). Teor de água e conteúdo de massa seca de embriões recém coletados. Valores médios acompanhados do desvio padrão.

Table 1 - Provenance of embryos of Inga vera subsp. affinis. Geographical position, climatic classification according to Köppen, maturation period and meteorological data, from flowering to collection (average of maximum and minimum air temperature, degree-day and precipitation). Water and dry matter content of freshly collected embryos. Values are average plus standard deviation.

\begin{tabular}{|c|c|c|c|c|c|c|}
\hline $\begin{array}{l}\text { Origem do material } \\
\text { Localização } \\
\text { e Classificação Climática }\end{array}$ & $\begin{array}{l}\text { Período fenológico } \\
\text { (total do ciclo) }\end{array}$ & $\begin{array}{l}\text { Max e } \\
\text { Min } \\
\left({ }^{\circ} \mathrm{C}\right)\end{array}$ & $\begin{array}{l}\text { Graus-dia } \\
\left({ }^{\circ} \mathrm{C} \mathrm{d}\right)\end{array}$ & $\begin{array}{c}\text { Chuva } \\
(\mathrm{mm})\end{array}$ & $\begin{array}{l}\text { Teor de água } \\
\left(\mathrm{g} \mathrm{g} \mathrm{g}^{-1}\right)\end{array}$ & $\begin{array}{l}\text { Massa seca } \\
\left(\text { g semente }^{-1}\right)\end{array}$ \\
\hline $\begin{array}{l}\text { Ribeirão Vermelho, MG (RIB) } \\
21^{\circ} 11^{\prime} \mathrm{S}, 45^{\circ} 03^{\prime} \mathrm{O} \text { e } 780 \mathrm{~m} \text {; Cwa }\end{array}$ & $\begin{array}{c}24 / 09 / 2009-09 / 01 / 2010 \\
(107 \text { dias })\end{array}$ & $29-18$ & 1485 & 652 & $1,39 \pm 0,21$ & $0,44 \pm 0,03$ \\
\hline $\begin{array}{l}\text { Piracicaba, SP (PIR) } \\
22^{\circ} 43^{\prime} \mathrm{S}, 47^{\circ} 38^{\prime} \mathrm{O} \text { e } 558 \mathrm{~m} \text {; Cwa }\end{array}$ & $\begin{array}{c}\text { 03/10/2009-07/01/2010 } \\
\text { (96 dias) }\end{array}$ & $30-19$ & 1404 & 532 & $1,66 \pm 0,08$ & $0,33 \pm 0,06$ \\
\hline $\begin{array}{l}\text { Vinhedo, SP (VIN) } \\
23^{\circ} 04^{\prime} \mathrm{S}, 47^{\circ} 01^{\prime} \mathrm{O} \text { e } 665 \mathrm{~m} \text {; Cwa }\end{array}$ & $\begin{array}{c}13 / 10 / 2008-13 / 01 / 2009 \\
(92 \text { dias })\end{array}$ & 29-18 & 1276 & 292 & $1,59 \pm 0,14$ & $0,29 \pm 0,01$ \\
\hline $\begin{array}{l}\text { São Paulo, SP (SAP2007) } \\
23^{\circ} 38^{\prime} \mathrm{S}, 46^{\circ} 37^{\prime} \mathrm{O} \text { e } 785 \mathrm{~m} \text {; Cwb }\end{array}$ & $\begin{array}{c}15 / 10 / 2006-31 / 01 / 2007 \\
(108 \text { dias })\end{array}$ & $27-17$ & 1303 & 676 & $1,34 \pm 0,03$ & $0,28 \pm 0,02$ \\
\hline $\begin{array}{l}\text { São Paulo, SP (SAP2008) } \\
23^{\circ} 38^{\prime} \mathrm{S}, 46^{\circ} 37^{\prime} \mathrm{O} \text { e } 785 \mathrm{~m} \text {; Cwb }\end{array}$ & $\begin{array}{c}15 / 10 / 2007-01 / 02 / 2008 \\
(109 \text { dias })\end{array}$ & $26-17$ & 1253 & 675 & $1,60 \pm 0,23$ & $0,22 \pm 0,02$ \\
\hline $\begin{array}{l}\text { São Paulo, SP (SAP2010) } \\
23^{\circ} 38^{\prime} \mathrm{S}, 46^{\circ} 37^{\prime} \mathrm{O} \text { e } 785 \mathrm{~m} \text {; Cwb }\end{array}$ & $\begin{array}{c}27 / 10 / 2009-19 / 02 / 2010 \\
\text { (115 dias) }\end{array}$ & 29-19 & 1605 & 1361 & $1,21 \pm 0,12$ & $0,26 \pm 0,01$ \\
\hline $\begin{array}{l}\text { Pariquera-Açú, SP (PAR) } \\
24^{\circ} 37^{\prime} \mathrm{S}, 47^{\circ} 53^{\prime} \mathrm{O} \text { e } 28 \mathrm{~m} \text {; Af }\end{array}$ & $\begin{array}{c}21 / 10 / 2009-04 / 02 / 2010 \\
(106 \text { dias })\end{array}$ & $31-22$ & 1739 & 896 & $1,50 \pm 0,24$ & $0,25 \pm 0,03$ \\
\hline
\end{tabular}

O teste de germinação foi conduzido em câmaras do tipo B.O.D., sob diferentes temperaturas $\left(10,15,20\right.$ e $\left.25^{\circ} \mathrm{C}\right)$, na presença de luz contínua, utilizando-se o método rolo de papel (papel Germitest $^{\mathbb{R}}$ ), ou seja, duas folhas para base e uma para cobertura (Bilia \& Barbedo 1997; Brasil 2009). As avaliações foram realizadas a cada dois dias, durante 35 dias, registrando-se os embriões que emitiram raiz primária (para o cálculo de germinação) e as que apresentaram capacidade de produção de plântulas normais (para o cálculo de crescimento de plântulas), seguindo descrições de Bilia \& Barbedo (1997) e Gurgel et al. (2012), sendo os resultados apresentados em porcentagem. Calculou-se, ainda, o índice de velocidade de germinação (IVG), com fórmula de Maguire (1962), o tempo médio de germinação e a variância do tempo médio de germinação (Borghetti \& Ferreira 2004).

$\mathrm{O}$ delineamento inteiramente casualizado em esquema fatorial $7 \times 4$ (origem do material $\mathrm{x}$ temperatura) foi utilizado, com três repetições de 20 embriões. Os dados obtidos foram submetidos à análise de variância (teste $\mathrm{F}$ ), ao nível de 5\% de significância, sem transformação, utilizandose o programa Excel $^{\circledR}$ (Ribeiro Junior 2005). Quando pertinente, as médias foram comparadas entre si pelo teste de Tukey, também ao nível de 5\% de significância (Santana \& Ranal 2004). Posteriormente, calcularam-se os coeficientes de correlação simples para todas as combinações entre os dados meteorológicos, teor de água, conteúdo de massa seca e germinação a $10,15,20$ e $25^{\circ} \mathrm{C}$, em que a significância dos valores de $\mathrm{r}$ foi determinada pelo teste $\mathrm{t}$, a $5 \%$ de probabilidade.

\section{Resultados e Discussão}

A duração do ciclo e os graus-dia acumulados no período de maturação das sementes em cada região e época, as temperaturas e a precipitação registradas no período e o teor de água e o conteúdo de massa seca dos embriões foram apresentados na Tabela 1. Comparando-se os três anos distintos das colheitas em São Paulo verifica-se que no ano de 2010 (SAP2010) o período de maturação foi cerca de uma semana mais longo que nos demais 
anos, recebendo precipitação praticamente duas vezes maior e acumulando cerca de 300 graus-dia a mais. Isso resultou em embriões com teor de água mais baixo na colheita, possivelmente indicando estarem mais maduros que os dos outros dois anos. Por outro lado, comparando-se diferentes regiões no mesmo ano (RIB, PIR, SAP2010 e PAR) verificam-se diferenças na maioria dos resultados de ciclo, graus-dia acumulados, precipitação e teor de água dos embriões. Contudo, verificaram-se correlações significativas (Tab. 2) somente entre ciclo e precipitação (positiva) e entre ciclo e teor de água (negativa).

A análise de variância (Tab. 3) para os dados de germinação, crescimento de plântulas, IVG, tempo médio e variância do tempo médio, apresentou interação significativa entre os fatores origem do material e temperatura (Tabs. 4, 5). A germinação dos embriões de $I$. vera subsp. affinis pode ocorrer a partir de $10^{\circ} \mathrm{C}$, mas depende das condições nas quais as sementes foram formadas, pois embriões de VIN e SAP2007 germinaram apenas a partir de $15^{\circ} \mathrm{C}$. Acima desta temperatura, os embriões de todas as origens apresentaram germinação superior a $90 \%$. Contudo, para a máxima expressão do crescimento de plântulas a exigência térmica dependeu da origem desses embriões, ora sendo de 15 a $25^{\circ} \mathrm{C}$ (PIR, VIN, SAP2007, SAP2010), ora de 20 a $25^{\circ} \mathrm{C}$ (RIB, SAP2008, PAR) (Tab. 4).
O IVG, o tempo médio de germinação e a variância do tempo médio também variaram em função da temperatura e da origem do material. Em geral, o aumento da temperatura promoveu aumento do IVG e diminuição do tempo médio, visto que o melhor desempenho em tempo, ou seja, maiores valores de IVG e menor de tempo médio, para algumas regiões, ocorreram a partir de $20^{\circ} \mathrm{C}$ e, para outras, a partir de $25^{\circ} \mathrm{C}$ (Tab. 5).

A análise sob diferentes temperaturas demonstrou que os embriões de $I$. vera subsp. affinis apresentam exigências térmicas distintas para a germinação, crescimento e desempenho em tempo, variando em função da origem do material. Contudo, verificou-se que a temperatura de $25^{\circ} \mathrm{C}$ apresentou-se adequada para os embriões de todas as origens. Tal temperatura está próxima à verificada por Bilia \& Barbedo (1997) para sementes dessa espécie, de uma única origem, e para sementes de outras espécies do mesmo gênero, como visto por Mendes-Rodrigues et al. (2007) para I. laurina (Sw.) Willd. e I. sessilis (Vell.) Mart. e por Nascimento et al. (2011) para I. ingoides (Rich.) Willd.

No presente estudo, as temperaturas subótimas (ou seja, abaixo de $25^{\circ} \mathrm{C}$ ) foram eficientes para identificar a influência do ambiente sobre as características germinativas. Por exemplo, a $10^{\circ} \mathrm{C}$ embriões oriundos de VIN e SAP2007 não

Tabela 2 - Coeficientes de correlação simples (r) entre os dados meteorológicos, teor de água, conteúdo de massa seca e germinação a 10, 15, 20 e $25^{\circ} \mathrm{C}$ de embriões de Inga vera subsp. affinis de diferentes origens. CICLO = ciclo de maturação; $\mathrm{MIN}$. = temperatura mínima; $\mathrm{MAX}$. = temperatura máxima; GD = graus-dia; $\mathrm{CHUVA}=$ chuva acumulada; $\mathrm{TA}=$ teor de água; $\mathrm{MS}=$ massa seca; $\mathrm{G}_{10}=$ germinação a $10^{\circ} \mathrm{C} ; \mathrm{G}_{15}=$ germinação a $15^{\circ} \mathrm{C} ; \mathrm{G}_{20}=$ germinação a $20^{\circ} \mathrm{C}$; $\mathrm{G}_{25}=$ germinação a $25^{\circ} \mathrm{C}$.

Table 2 - Coefficient of simple correlation (r) among meteorological data, water content, dry mass and germination at 10, 15, 20 e $25^{\circ} \mathrm{C}$ of embryos of Inga vera subsp. affinis from different regions. CICLO = maturation period; MIN. = minimum temperature; MAX. $=$ maximum temperature; $\mathrm{GD}=$ degree-day; $\mathrm{CHUVA}=$ accumulated rainfall; $\mathrm{TA}=$ water content $\mathrm{MS}=$ dry mass; $\mathrm{G}_{10}=$ germination at $10^{\circ} \mathrm{C} ; \mathrm{G}_{15}=$ germination at $15^{\circ} \mathrm{C} ; \mathrm{G}_{20}=$ germination at $20^{\circ} \mathrm{C} ; \mathrm{G}_{25}=$ germination at $25^{\circ} \mathrm{C}$.

\begin{tabular}{|c|c|c|c|c|c|c|c|c|c|c|}
\hline & MAX. & MIN. & GD & CHUVA & $\mathbf{T A}$ & MS & $G_{10}$ & $\mathbf{G}_{15}$ & $G_{20}$ & $\mathbf{G}_{25}$ \\
\hline Ciclo & $-0,31^{\mathrm{NS}}$ & $-0,01^{\mathrm{NS}}$ & $0,38^{\mathrm{NS}}$ & $0,84 *$ & $-0,76^{*}$ & $-0,20^{\mathrm{NS}}$ & $0,18^{\mathrm{NS}}$ & $-0,36^{\mathrm{NS}}$ & $-0,63^{\mathrm{NS}}$ & $0,60^{\mathrm{NS}}$ \\
\hline Max. & & $0,86^{*}$ & $0,74^{\mathrm{NS}}$ & $0,12^{\mathrm{NS}}$ & $0,07^{\mathrm{NS}}$ & $0,27^{\mathrm{NS}}$ & $0,76^{*}$ & $0,58^{\mathrm{NS}}$ & $0,75^{*}$ & $0,26^{\mathrm{NS}}$ \\
\hline Min. & & & $0,88^{*}$ & $0,35^{\mathrm{NS}}$ & $0,03^{\mathrm{NS}}$ & $-0,12^{\mathrm{NS}}$ & $0,73^{\mathrm{NS}}$ & $0,17^{\mathrm{NS}}$ & $0,53^{\mathrm{NS}}$ & $0,39^{\mathrm{NS}}$ \\
\hline GD & & & & $0,67^{\mathrm{NS}}$ & $-0,39^{\mathrm{NS}}$ & $0,03^{\mathrm{NS}}$ & $0,82 *$ & $0,22^{\mathrm{NS}}$ & $0,31^{\mathrm{NS}}$ & $0,63^{\mathrm{NS}}$ \\
\hline Chuva & & & & & $-0,75^{\mathrm{NS}}$ & $-0,28^{\mathrm{NS}}$ & $0,52^{\mathrm{NS}}$ & $-0,01^{\mathrm{NS}}$ & $-0,39^{\mathrm{NS}}$ & $0,63^{\mathrm{NS}}$ \\
\hline TA & & & & & & $-0,08^{\mathrm{NS}}$ & $-0,15^{\mathrm{NS}}$ & $-0,04^{\mathrm{NS}}$ & $0,52^{\mathrm{NS}}$ & $-0,33^{\mathrm{NS}}$ \\
\hline MS & & & & & & & $0,28^{\mathrm{NS}}$ & $0,58^{\mathrm{NS}}$ & $0,48^{\mathrm{NS}}$ & $0,20^{\mathrm{NS}}$ \\
\hline $\mathrm{G}_{10}$ & & & & & & & & $0,49^{\mathrm{NS}}$ & $0,46^{\mathrm{NS}}$ & $0,77^{*}$ \\
\hline $\mathrm{G}_{15}$ & & & & & & & & & $0,62^{\mathrm{NS}}$ & $-0,03^{\mathrm{NS}}$ \\
\hline $\mathrm{G}_{20}$ & & & & & & & & & & $-0,05^{\mathrm{NS}}$ \\
\hline
\end{tabular}


Tabela 3 - Valores de F e nível de significância das interações entre origem do material e temperatura de germinação, obtidos na análise de variância de embriões de Inga vera subsp. affinis.

Table 3-Values of $\mathrm{F}$ and significance levels of the interactions between origin of the material and germination temperature, obtained from analysis of variance of embryos of Inga vera subsp. affinis.

\begin{tabular}{lcc}
\hline Variável & Valor de F & Nível de significância \\
\hline $\begin{array}{l}\text { Germinação } \\
\text { Crescimento } \\
\text { de plântulas }\end{array}$ & 260,79 & $<0,0001$ \\
IVG & 69,79 & $<0,0001$ \\
Tempo médio & 341,41 & $<0,0001$ \\
$\begin{array}{l}\text { Variância } \\
\text { do tempo médio }\end{array}$ & 8,58 & $<0,0001$ \\
\hline
\end{tabular}

germinaram, enquanto que os oriundos de RIB, PIR, SAP2010 e PAR apresentaram elevadas taxas de germinação (Tab. 4). Essas diferenças permaneceram para embriões oriundos da mesma matriz, mas de épocas distintas, como visto para SAP2007, SAP2008 e SAP2010 apresentando, respectivamente, $0 \%, 9 \%$ e $92 \%$ de germinação (Tab. 4). Quando consideradas as demais variáveis (crescimento de plântulas, IVG e tempo médio), as diferenças entre as origens dos embriões ocorreram para as outras temperaturas $\left(15,20\right.$ e $\left.25^{\circ} \mathrm{C}\right) . \mathrm{O}$ crescimento de plântulas diferiu, principalmente, a $15^{\circ} \mathrm{C}$, sendo menores valores para as regiões de RIB e SAP2008 (Tab. 4). No IVG, valores que para RIB, PIR, SAP2007, SAP2010 e PAR ocorreram a $15^{\circ} \mathrm{C}$, para SAP2008 e VIN ocorreram, respectivamente, a $20^{\circ} \mathrm{C}$ e $25^{\circ} \mathrm{C}$ e no tempo médio, valores que para PIR e SAP2008 ocorreram a $15^{\circ} \mathrm{C}$ para SAP2007 ocorreu apenas a $20^{\circ} \mathrm{C}$ (Tab. 5).

As condições hídricas e térmicas, registradas durante o desenvolvimento e maturação dos embriões de Inga vera subsp. affinis (Tab. 1), podem ter influenciado o seu comportamento germinativo, principalmente nas baixas temperaturas e quando há diferença maior que $100^{\circ} \mathrm{C}$.dia para a formação das sementes. Quando comparadas às regiões de PIR e VIN, por exemplo, quanto à germinação a $10^{\circ} \mathrm{C}$ e ao acúmulo de graus dia $\left(\mathrm{G}_{10} \times \mathrm{GD}\right.$, Tab. 2$)$ verifica-se que o somatório de graus-dia foi maior para os embriões que germinaram nessa temperatura (Tabs. 1 e 4). Entre as regiões de SAP2010 e VIN, na qual os embriões também diferiram sob $10^{\circ} \mathrm{C}$ (Tab. 4) observou-se novamente diferença no acúmulo de graus-dia (maiores valores para SAP2010). Notase, ainda, que para SAP2010 a duração do ciclo de maturação foi maior e os embriões dispersos com teor de água mais baixo (Tab. 1).

Respostas semelhantes ocorreram entre as colheitas na mesma região em épocas distintas (SAP), na qual a época que proporcionou maior quantidade de graus-dia e chuva acumulada teve os embriões dispersos mais maduros (visto pelo teor de água) e com melhor desempenho em tempo e maior porcentagem de germinação sob baixas temperaturas (Tabs. 1, 4, 5). Para regiões cujos embriões expressaram germinação a $10^{\circ} \mathrm{C}$ (valores maiores que $70 \%$ ), mas cujas médias diferiram significativamente, como RIB e PAR, verificou-se também maior valor de germinação para aquela que recebeu mais graus-dia durante a maturação (Tabs. 1, 4).

Estudos relatam que diferenças nas características fisiológicas e germinativas em sementes de populações da mesma espécie podem ser resultantes do grau de maturidade ou da qualidade inicial das sementes, condicionadas pelas variáveis meteorológicas do ambiente durante a formação, como a precipitação pluvial e a temperatura do ar (Daws et al. 2004, 2006; Martins et al. 2009; Lamarca et al. 2011; Pereira et al. 2012; Lamarca et al. 2013). Tal fato também pode ter ocorrido para os embriões de Inga vera subsp. affinis do presente trabalho. Para Daws et al. (2004, 2006), a temperatura do ar durante o desenvolvimento e maturação das sementes (considerada pelo acúmulo de graus-dia) influencia a continuidade do desenvolvimento, condicionando diversas características físicas e fisiológicas, tais como o teor de água inicial e o acúmulo de massa seca, os níveis de dormência e de tolerância à dessecação, bem como a temperatura basal e a exigência térmica para germinação. Além disso, a temperatura do ar pode regular a expressão de genes envolvidos em processos fisiológicos, como a fenologia, o desenvolvimento e a germinação, estando os graus-dia fortemente associados com o amadurecimento de sementes (Johnsen et al. 2005). Essa ação da temperatura foi demonstrada para outras espécies. Lamarca et al. (2011), por exemplo, verificaram que houve maior diferença na exigência térmica para germinação em sementes de Eugenia pyriformis Cambess. 
Tabela 4-Germinação e crescimento de plântula normal de embriões de Inga vera subsp. affinis de origens distintas, sob diferentes temperaturas. Médias seguidas pela mesma letra (minúsculas nas linhas, maiúsculas nas colunas) não diferem entre si pelo teste de Tukey, a 5\%.

Table 4 - Germination and development of normal seedling of embryos of Inga vera subsp. affinis from different provenances and at different temperatures. Means followed by the same letter (lowercase in the lines, capitals in the columns) do not differ by Tukey test at $5 \%$.

Origem do material

Temperatura $\left({ }^{\circ} \mathrm{C}\right)$

$10 \quad 15 \quad 20 \quad 25$

Germinação (\%)

Ribeirão Vermelho (RIB)

Piracicaba (PIR)

Vinhedo (VIN)

São Paulo (SAP2007)

São Paulo (SAP2008)

São Paulo (SAP2010)

Pariquera-Açú (PAR)

$75 \mathrm{bC}$

$100 \mathrm{aA}$

$100 \mathrm{aA}$

$100 \mathrm{aA}$

$100 \mathrm{aA}$

$100 \mathrm{aA}$

$100 \mathrm{aA}$

$100 \mathrm{aA}$

$0 \mathrm{cE}$

$100 \mathrm{aA}$

$100 \mathrm{aA}$

$93 \mathrm{bB}$

$0 \mathrm{bE}$

$97 \mathrm{aA}$

97 aA

$98 \mathrm{aAB}$

$9 \mathrm{bD}$

$97 \mathrm{aA}$

$98 \mathrm{aA}$

$98 \mathrm{aAB}$

$92 \mathrm{bB}$

$100 \mathrm{aA}$

$98 \mathrm{aA}$

$100 \mathrm{aA}$

$97 \mathrm{aAB}$

98 aA

$100 \mathrm{aA}$

$100 \mathrm{aA}$

Coeficiente de variação (\%)

2,86

Crescimento de plântulas normais (\%)

Ribeirão Vermelho (RIB)

$0 \mathrm{cA}$

$60 \mathrm{bC}$

$82 \mathrm{aB}$

$92 \mathrm{aA}$

Piracicaba (PIR)

$0 \mathrm{bA}$

93 aAB

98 aA

98 aA

Vinhedo (VIN)

$0 \mathrm{bA}$

$100 \mathrm{aA}$

98 aA

92 aA

São Paulo (SAP2007)

$0 \mathrm{bA}$

89 aAB

$93 \mathrm{aAB}$

94 aA

$0 \mathrm{cA}$

$60 \mathrm{bC}$

$95 \mathrm{aA}$

98 aA

$0 \mathrm{bA}$

$90 \mathrm{aAB}$

$93 \mathrm{aAB}$

98 aA

$0 \mathrm{cA}$

83 bB

$98 \mathrm{aA}$

$98 \mathrm{aA}$

Coeficiente de variação (\%)

(Myrtaceae) oriundas de diferentes regiões do que sementes de diferentes espécies de Eugenia L. oriundas da mesma região e época, sugerindo que o comportamento germinativo pode ser decorrente de adaptações às condições térmicas do local de origem. Esses autores observaram, também, que as principais diferenças nessa exigência, entre as populações de Eugenia pyriformis, ocorreram para as temperaturas extremas. Mattana et al. (2012) também verificaram que sementes da mesma espécie (Aquilegia barbaricina Arrigoni \& E. Nardi e Aquilegia nugorensis Arrigoni \& E. Nardi), oriundas de populações diferentes, separadas por barreiras geográficas e formadas sob diferentes condições ambientais, apresentam respostas germinativas distintas sob diferentes temperaturas.

Em embriões de Inga vera subsp. affinis, respostas fisiológicas distintas já haviam sido observadas para os limites de tolerância à dessecação e para o potencial de armazenamento dos embriões (Bilia et al. 1998; Bilia et al. 1999; Barbedo \& Cicero 2000; Faria et al. 2004; Andréo et al. 2006; Bonjovani \& Barbedo 2008; Parisi et al. 2013), reforçando a ideia que as variações fisiológicas encontradas entre os embriões de $I$. 
Tabela 5 - Índice de velocidade de germinação, tempo médio de germinação e variância do tempo médio de germinação de embriões de Inga vera subsp. affinis de origens distintas, sob diferentes temperaturas. Médias seguidas pela mesma letra (minúsculas nas linhas, maiúsculas nas colunas) não diferem entre si pelo teste de Tukey, a 5\%.

Table 5 - Index germination rate, mean germination time and variance of the average germination time of embryos of Inga vera subsp. affinis from different sources and at different temperatures. Means followed by the same letter (lowercase on the lines, capitals on the columns) do not differ by Tukey test at $5 \%$.

\begin{tabular}{|c|c|c|c|c|}
\hline \multirow{2}{*}{ Origem do material } & \multicolumn{4}{|c|}{ Temperatura $\left({ }^{\circ} \mathrm{C}\right)$} \\
\hline & 10 & 15 & 20 & 25 \\
\hline \multicolumn{5}{|c|}{ Índice de velocidade de germinação } \\
\hline Ribeirão Vermelho (RIB) & $0,9 \mathrm{dA}$ & $1,9 \mathrm{cAB}$ & $2,9 \mathrm{bcD}$ & $4,2 \mathrm{aE}$ \\
\hline Piracicaba (PIR) & $1,3 \mathrm{dA}$ & $2,2 \mathrm{cA}$ & $3,7 \mathrm{bB}$ & $4,9 \mathrm{aD}$ \\
\hline Vinhedo (VIN) & $0,0 \mathrm{cB}$ & $1,1 \mathrm{bC}$ & $1,8 \mathrm{aE}$ & $2,0 \mathrm{aF}$ \\
\hline São Paulo (SAP2007) & $0,0 \mathrm{~dB}$ & $2,2 \mathrm{cA}$ & $5,3 \mathrm{bA}$ & $7,8 \mathrm{aA}$ \\
\hline São Paulo (SAP2008) & $0,1 \mathrm{~dB}$ & $1,6 \mathrm{cB}$ & $2,5 \mathrm{bD}$ & $4,1 \mathrm{aE}$ \\
\hline São Paulo (SAP2010) & $0,9 \mathrm{dA}$ & $2,0 \mathrm{cAB}$ & $3,3 \mathrm{bBC}$ & $6,5 \mathrm{aB}$ \\
\hline Pariquera-Açú (PAR) & $1,1 \mathrm{dA}$ & $2,3 \mathrm{cA}$ & $3,6 \mathrm{bB}$ & $5,7 \mathrm{aC}$ \\
\hline Coeficiente de variação (\%) & \multicolumn{4}{|c|}{6,67} \\
\hline \multicolumn{5}{|l|}{ Tempo médio de germinação } \\
\hline Ribeirão Vermelho (RIB) & $18,4 \mathrm{aC}$ & $11,0 \mathrm{bB}$ & $7,4 \mathrm{cC}$ & $4,9 \mathrm{dC}$ \\
\hline Piracicaba (PIR) & $15,9 \mathrm{aD}$ & $9,5 \mathrm{bBC}$ & $5,6 \mathrm{cD}$ & $4,2 \mathrm{cC}$ \\
\hline Vinhedo (VIN) & $0,0 \mathrm{cE}$ & $19,3 \mathrm{aA}$ & $11,4 \mathrm{bA}$ & $10,1 \mathrm{bA}$ \\
\hline São Paulo (SAP2007) & $0,0 \mathrm{dE}$ & $10,9 \mathrm{aB}$ & $9,5 \mathrm{bB}$ & $6,7 \mathrm{cB}$ \\
\hline São Paulo (SAP2008) & $30,3 \mathrm{aA}$ & $9,5 \mathrm{bBC}$ & $6,1 \mathrm{cD}$ & $3,9 \mathrm{dC}$ \\
\hline São Paulo (SAP2010) & $22,9 \mathrm{aB}$ & $10,4 \mathrm{bB}$ & $6,3 \mathrm{cCD}$ & $3,4 \mathrm{dC}$ \\
\hline Pariquera-Açú (PAR) & $18,8 \mathrm{aC}$ & $8,6 \mathrm{bC}$ & $5,8 \mathrm{cCD}$ & $3,6 \mathrm{dC}$ \\
\hline Coeficiente de variação (\%) & \multicolumn{4}{|c|}{6,53} \\
\hline \multicolumn{5}{|l|}{ Variância do tempo médio } \\
\hline Ribeirão Vermelho (RIB) & $30,6 \mathrm{aA}$ & $5,8 \mathrm{bB}$ & $2,0 \mathrm{bB}$ & $0,7 \mathrm{bA}$ \\
\hline Piracicaba (PIR) & $4,8 \mathrm{aBC}$ & $4,2 \mathrm{aB}$ & $0,9 \mathrm{aB}$ & $1,2 \mathrm{aA}$ \\
\hline Vinhedo (VIN) & $0,0 \mathrm{aC}$ & $8,1 \mathrm{aB}$ & 7,9 aAB & $5,3 \mathrm{aA}$ \\
\hline São Paulo (SAP2007) & $0,0 \mathrm{aA}$ & $4,0 \mathrm{aB}$ & $5,6 \mathrm{aAB}$ & $4,0 \mathrm{aA}$ \\
\hline São Paulo (SAP2008) & $0,7 \mathrm{cC}$ & $72,1 \mathrm{aA}$ & $25,4 \mathrm{bA}$ & $19,9 \mathrm{bA}$ \\
\hline São Paulo (SAP2010) & $21,9 \mathrm{aAB}$ & $3,7 \mathrm{bB}$ & $2,2 \mathrm{bB}$ & $1,2 \mathrm{bA}$ \\
\hline Pariquera-Açú (PAR) & $10,9 \mathrm{aABC}$ & $0,9 \mathrm{aB}$ & $1,0 \mathrm{aB}$ & $0,5 \mathrm{aA}$ \\
\hline Coeficiente de variação (\%) & \multicolumn{4}{|c|}{93,00} \\
\hline
\end{tabular}


vera subsp. affinis podem estar relacionadas com a procedência do material, com a qualidade inicial e com o grau de maturidade que são dispersos. Aliados aos resultados obtidos no presente estudo verifica-se que as respostas germinativas de embriões de I. vera subsp. affinis são decorrentes da origem do material e podem estar associadas ao genótipo das populações, às condições do ambiente de formação e ao grau de maturidade das sementes no momento da dispersão natural e colheita dos frutos.

Os resultados obtidos no presente trabalho permitiram concluir que a germinação de embriões de I. vera subsp. affinis sob diferentes temperaturas é dependente da origem do material, podendo estar relacionada com as variações hídricas e térmicas do ambiente durante o desenvolvimento e maturação. As principais diferenças germinativas entre as origens dos materiais ocorrem sob baixas temperaturas e, dependendo da origem do material, os embriões de I. vera subsp. affinis podem germinar a partir de $10^{\circ} \mathrm{C}$ ou de $15^{\circ} \mathrm{C}$.

\section{Agradecimentos}

Os autores agradecem ao Dr. Domingos Sávio Rodrigues (IBt), ao Dr. João José Dias Parisi (IAC), à Prefeitura de Piracicaba - SP, à CCR AutoBAn, ao Centro de Exposições Imigrantes $\mathrm{SP}$ e ao Pólo Regional Vale do Ribeira, Pariqueraaçú - SP, a permissão e auxilio nas colheitas de frutos e sementes; à Dra. Lucia Rossi (IBt), a identificação da espécie; à Universidade Federal de Lavras, Lavras, MG; ao Instituto Agronômico de Campinas, Campinas, SP e ao Instituto Astronômico e Geofísico da Universidade de São Paulo, São Paulo, SP, o fornecimento dos dados meteorológicos; à CAPES, a bolsa concedida a E.V. Lamarca (Doutorado) e ao CNPq, as bolsas concedidas a C.J. Barbedo e a J.M.R. Faria (produtividade em pesquisa) e o auxílio financeiro ao projeto (Proc.477640/2009-5).

\section{Referências}

Andréo, Y.; Nakagawa, J. \& Barbedo, C.J. 2006. Mobilização de água e conservação da viabilidade de embriões de sementes recalcitrantes de ingá (Inga vera Will. subsp. affinis (DC.) T. D. Penn.). Revista Brasileira de Botânica 29: 309-318.

Barbedo, C.J. \& Cicero, S.M. 2000. Effects of initial quality, low temperature and $\mathrm{ABA}$ on the storage of seeds of Inga uruguensis, a tropical species with recalcitrant seeds. Seed Science and Technology 28: 793-808.
Bilia, D.A.C. \& Barbedo, C.J. 1997. Estudos de germinação e armazenamento de sementes de Inga uruguensis Hook. et Arn. Científica 25: 379-391.

Bilia, D.A.C.; Marcos Filho, J. \& Novembre, A.D.C.L. 1998. Conservação da qualidade fisiológica de sementes de Inga uruguensis Hook. et Arn. Revista Brasileira de Sementes 20: 48-54.

Bilia, D.A.C.; Marcos Filho, J. \& Novembre, A.D.C.L. 1999. Desiccation tolerance and seed storability of Inga uruguensis Hook. et Arn. Seed Science and Technology 27: 77-89.

Bilia, D.A.C.; Barbedo, C.J.; Cicero, S.M. \& Marcos Filho, J. 2003. Ingá: uma espécie importante para recomposição vegetal em florestas ripárias, com sementes interessantes para a ciência. Informativo Abrates 13: 26-30.

Bonjovani, M.J. \& Barbedo, C.J. 2008. Sementes recalcitrantes: intolerantes a baixas temperaturas? Embriões recalcitrantes de Inga vera Willd. subsp. affinis (DC.) T. D. Penn. toleram temperatura subzero. Revista Brasileira de Botânica 31: 345-356.

Borghetti, F. 2005. Temperaturas extremas e a germinação das sementes. In: Nogueira, R.M.C. et al. (orgs.). Estresses ambientais: danos e benefícios em plantas. Imprensa Universitária, Universidade Federal Rural de Pernambuco, Recife. Pp. 207-218.

Borguetti, F. \& Ferreira, A.G. 2004. Interpretação de resultados de germinação. In: Ferreira, A.G. \& Borghetti, F. (orgs.). Germinação: do básico ao aplicado. Artmed, Porto Alegre. Pp. 209-222.

Brasil. 2009. Regras para análise de sementes. Ministério da Agricultura, Pecuária e Abastecimento, Brasília. 399p.

Daws, M.I.; Cleland, H.; Chmielarz, P.; Gorin, F.; Leprince, O.; Matthews, S.; Mullins, C.E.; Thanos, C.A.; Vandvik, V. \& Pritchard, H.W. 2006. Variable dessication tolerance in Acer pseudoplatanus seeds in relation to developmental conditions: a case of phenotypic recalcitrance? Functional Plant Biology 33: 59-66.

Daws, M.I.; Lydall, E.; Chmielarz, P.; Leprince, O.; Matthews, S.; Thanos, C.A. \& Pritchard, H.W. 2004. Developmental heat sum influences recalcitrant seed traits in Aesculus hippocastanum across Europe. New Phytologist 162: 157-166.

Delgado, L.F. \& Barbedo, C.J. 2012. Water potential and viability of seeds of Eugenia (Myrtaceae), a tropical tree species, based upon different levels of drying. Brazilian Archives of Biology and Technology 55: 583-590.

Faria, J.M.R.; Lammeren, A.A.M.V. \& Hilhorst, H.W.M. 2004. Desiccation sensitivity and cell cycle aspects in seeds of Inga vera subsp. Affinis. Seed Science Research 14: 165-178.

Figliolia. M.B. \& Kageyama, P.Y. 1994. Maturação de sementes de Inga uruguensis Hook. et Arn. em 
floresta ripária do rio Moji-Guaçu, Município de Moji-Guaçu. Revista do Instituto Florestal 6: 13-52.

Gurgel, E.S.C.; Santos, J.U.M.; Lucas, F.C.A. \& Bastos, M.N.C.B. 2012. Morfologia de plântulas de Leguminosae e o potencial sistemático. Rodriguésia 63: 65-73.

Johnsen, Ø.; Fossdal, C.G.; Nagy, N.; Mølmann, J.; Dæhlen, O.G. \& Skrøppa, T. 2005. Climatic adaptation in Picea abies progenies is affected by the temperature during zygotic embryogenesis and seed maturation. Plant, Cell and Environment 28: 1090-1102.

Joly, A.B. 1993. Botânica: introdução à taxonomia vegetal. $11^{\mathrm{a}}$ ed. Ed. Nacional, São Paulo. 777p.

Lamarca, E.V.; Prataviera, J.S.; Borges, I.F.; Delgado, L.F.; Teixeira, C.C.; Camargo, M.B.P.; Faria, J.M.R. \& Barbedo, C.J. 2013. Maturation of Eugenia pyriformis seeds under different hydric and thermal conditions. Anais da Academia Brasileira de Ciências 85: 223-233.

Lamarca, E.V.; Silva, C.V. \& Barbedo, C.J. 2011. Limites térmicos para a germinação em função da origem de sementes de espécies de Eugenia (Myrtaceae) nativas do Brasil. Acta Botanica Brasilica 25: 293-300.

Maguire, J.D. 1962. Speed of germination-aid in selection and evaluation for seedling emergence and vigor. Crop Science 2: 176-177.

Martins, C.C.; Bovi, M.L.A.; Nakagawa, J. \& Machado, C.G. 2009. Secagem e armazenamento de sementes de juçara. Revista Árvore 33: 635-642.

Mattana, E.; Daws, M.I.; Fenu, G. \& Bacchetta, G. 2012. Adaptation to habitat in Aquilegia species endemic to Sardinia (Italy): Seed dispersal, germination and persistence in the soil. Plant Biosystems 146: 374-383.

Mello, J.I.O. \& Barbedo, C.J. 2007. Temperatura, luz e substrato para a germinação de sementes de paubrasil Caesalpinia echinata Lam., LeguminosaeCaesalpiniodeae. Revista Árvore 31: 645-655.

Mendes-Rodrigues, C.; Ferreira, W.R.; Lima, J.A.; Dornelles, M.C.; Ranal, M. \& Santana, D,G. 2007. Germinação de embriões de duas espécies de Inga (Mimosaceae). Revista Brasileira de Biociências 5: 561-563.

Nascimento, I.L.; Leal, C.C.P.; Nogueira, N.W.; Medeiros, A.K.P. \& Câmara, F.M.M. 2011. Influência de diferentes tipos de substrato e temperatura na germinação de sementes de Inga ingoides (Rich.) Willd. Revista Verde de Agroecologia e Desenvolvimento Sustentável 6: 7-10.

Parisi, J.J.D.; Biagi, J.D.; Barbedo, J.C. \& Medina, P.F. 2013. Viability of Inga vera Willd. subsp. affinis (DC.) T. D. Penn. Embryos according to the maturation stage, fungal incidence, chemical treatment and storage. Journal of Seed Science 35: 70-76.

Pascuali, L.C.; Silva, F.S.; Porto, A.G.; Silva Filho, A. \& Meneghello, G.E. 2012. Germinação de sementes de pinhão manso em diferentes temperaturas, luz e substratos. Semina: Ciências Agrárias 33: 1435-1440.

Pedro Junior, M.J.; Brunini, O.; Alfonsi, R.R. \& Angelocci, L.R. 1977. Estimativa de graus-dia em função de altitude e latitude para o estado de São Paulo. Bragantia 36: 89-92.

Pereira, W.V.S.; Faria, J.M.R.; Tonetti, O.A.O. \& Silva, E.A.A. 2012. Desiccation tolerance of Tapirira obtusa seeds collected from different environments. Revista Brasileira de Sementes 34: 388-396.

Pimenta, R.S.; Luz, P.B.; Pivetta, K.F.L.; Castro, A. \& Pizetta, P.U.C. 2010. Efeito da maturação e temperatura na germinação de sementes de Phoenix canariensis hort. ex Chabaud - Arecaceae. Revista Árvore 34: 31-38.

Pires, L.A.; Cardoso, V.J.M.; Joly, C.A. \& Rodrigues, R.R. 2009. Germinação de Ternstroemia brasiliensis Cambess. (Pentaphylacaceae) de Floresta de Restinga. Acta Botanica Brasilica 23: 57-66.

Ribeiro Junior, J.I. 2005. Análises estatísticas no Excel: guia prático. Editora da Universidade Federal de Viçosa, Viçosa. 251p.

Santana, D.G \& Ranal, M.A. 2004. Análise da germinação: um enfoque estatístico. Universidade de Brasília, Brasília. 247p.

Stockman, A.L.; Brancalion, P.H.S.; Novembre, A.D.L.C. \& Chamma, H.M.C.P. 2007. Sementes de ipê-branco (Tabebuia roseo-alba (Ridl.) Sand. - Bignoniaceae): temperatura e substrato para o teste de germinação. Revista Brasileira de Sementes 29: 139-143.

Villa Nova, N.A.; Pedro Júnior, M.J.; Pereira, A.R. \& Ometto, J.C. 1972. Estimativa de graus-dia acumulados acima de qualquer temperatura base em função das temperaturas máxima e mínima. Caderno Ciência da Terra 30: 89-92. 